\title{
CENTRIC MAPS AND REALIZATION OF DIAGRAMS IN THE HOMOTOPY CATEGORY
}

\author{
W. G. DWYER AND D. M. KAN
}

(Communicated by Frederick R. Cohen)

\begin{abstract}
We study the problem of taking a diagram in the homotopy category and realizing it as a diagram in the category of spaces. Under some assumptions there is a relatively simple obstruction theory for doing this; these assumptions hold in two cases involving homotopy decomposition diagrams for the classifying spaces of compact Lie groups.
\end{abstract}

\section{INTRODUCTION}

Let $\mathbf{D}$ be a small category. Suppose that $\bar{X}$ is a D-diagram in the homotopy category (in other words, a functor from $\mathbf{D}$ to the homotopy category of simplicial sets). The question of whether or not $\bar{X}$ can be realized by a Ddiagram of simplicial sets has been treated by [5]. The purpose of this note is to study a special situation in which the treatment can be simplified quite a bit. We look at two examples to which this simplified treatment is applicable; both examples involve homotopy decomposition diagrams for compact Lie groups. Our results show that in at least one of these examples [13] the decomposition diagram is completely determined by its underlying diagram in the homotopy category. It is possible that this "rigidity" result will eventually contribute to a general homotopy theoretic characterization theorem for classifying spaces of compact Lie groups (cf. [8]).

Before going any further we have to introduce some notation. The symbol $\mathbf{S}$ will denote the category of simplicial sets and ho $\mathbf{S}$ the associated homotopy category obtained by localizing with respect to (i.e., formally inverting) weak equivalences. If $\mathbf{C}$ is a category and $\mathbf{D}$ is a small category, then $\mathbf{C}^{\mathbf{D}}$ will stand for the category of D-diagrams in $\mathbf{C}$; the objects of $\mathbf{C}^{\mathbf{D}}$ are functors $\mathbf{D} \rightarrow \mathbf{C}$ and the maps of $\mathbf{C}^{\mathbf{D}}$ are natural transformations. There is a projection functor $\pi: \mathbf{S} \rightarrow$ ho $\mathbf{S}$; we will use the same symbol for induced functors $\mathbf{S}^{\mathbf{D}} \rightarrow(\text { ho } \mathbf{S})^{\mathbf{D}}$. Both $\mathbf{S}$ and $\mathbf{S}^{\mathbf{D}}$ admit closed simplicial model category structures [19, II; 20, p. $233 ; 4, \S 2]$, and we will sometimes require without loss of generality that chosen objects in these categories be fibrant.

Given a small category $\mathbf{D}$ and a diagram $\bar{X}$ in $(\text { ho } \mathbf{S})^{\mathbf{D}}$, a realization of $\bar{X}[5,3.1]$ is a pair $(Y, f)$ such that $Y$ is an object of $\mathbf{S}^{\mathbf{D}}$ and $f$ is an

Received by the editors August 9, 1990.

1980 Mathematics Subject Classification (1985 Revision). Primary 55D15; Secondary 55F35.

This work was supported in part by the National Science Foundation.

(C) 1992 American Mathematical Society $0002-9939 / 92 \$ 1.00+\$ .25$ per page 
isomorphism $f: \pi Y \cong \bar{X}$ in (ho $\mathbf{S})^{\mathbf{D}}$. A weak equivalence between two such realizations $\left(Y, f_{Y}\right)$ and $\left(Z, f_{Z}\right)$ is a weak equivalence $h: Y \rightarrow Z \in \mathbf{S}^{\mathbf{D}}$ such that $f_{Z} \circ(\pi h)=f_{Y}$.

If $f: A \rightarrow B$ is a map of simplicial sets, let $\operatorname{Map}(A, B)_{f}$ denote the component of the simplicial mapping space $\operatorname{Map}(A, B)$ containing $f$ [17, p. 17]. Call a map $f: A \rightarrow B$ between fibrant simplicial sets centric if precomposition with $f$ induces a homotopy equivalence $\operatorname{Map}(A, A)_{1} \rightarrow \operatorname{Map}(A, B)_{f}$. Here "1" stands for the identity map of $A$ and $\operatorname{Map}(A, A)_{1}$, also denoted haut $(A)_{1}$, is the identity component of the simplicial monoid of homotopy self-equivalences of $A$. It is clear that whether or not a map is centric is a homotopy property. We will say that a homotopy class of maps $\phi: A \rightarrow B$ is centric if any representative is, and that a diagram $X$ in $\mathbf{S}^{\mathbf{D}}$ (resp. $\bar{X}$ in $(\text { ho } \mathbf{S})^{\mathbf{D}}$ ) is centric if $X(g)$ (resp. $\bar{X}(g)$ ) is centric for each morphism $g$ of $\mathbf{D}$.

Remark. A map $i: G \rightarrow H$ of discrete groups induces a centric map $B G \rightarrow B H$ of classifying spaces iff $i$ induces an isomorphism between the center of $G$ and the centralizer in $H$ of $i(G)$. This explains the choice of the term "centric."

Given a centric diagram $\bar{X}$ in (ho $\mathbf{S})^{\mathbf{D}}$ construct functors $\alpha_{i} \bar{X}: \mathbf{D}^{\text {op }} \rightarrow \mathbf{A b}$ (where $\mathbf{A b}$ is the category of abelian groups) by setting $\alpha_{i} \bar{X}(D)=\pi_{i}$ haut $(\bar{X}(D))_{1}$ for $D$ an object of $\mathbf{D}$; there is no basepoint problem with these homotopy groups because haut $(\bar{X}(D))_{1}$ is a simple space. For each map $g: D_{0} \rightarrow D_{1}$ in D the induced map $\alpha_{i} \bar{X}(g): \alpha_{i} \bar{X}\left(D_{1}\right) \rightarrow \alpha_{i} \bar{X}\left(D_{0}\right)$ is the composite

$$
\pi_{i} \operatorname{haut}\left(\bar{X}\left(D_{1}\right)\right)_{1} \stackrel{\gamma_{\mathrm{t}}}{\longrightarrow} \pi_{i} \operatorname{Map}\left(\bar{X}\left(D_{0}\right), \bar{X}\left(D_{1}\right)\right)_{\gamma} \stackrel{\left(\gamma^{\sharp}\right)^{-1}}{\longrightarrow} \pi_{i} \operatorname{haut}\left(\bar{X}\left(D_{0}\right)\right)_{1},
$$

where $\gamma$ is a representative of $\bar{X}(g), \gamma_{\sharp}$ is induced by postcomposition with $\gamma$ and $\gamma^{\sharp}$ by precomposition.

Our main technical result is the following one:

Theorem 1.1. Suppose that $\mathbf{D}$ is a small category and that $\bar{X} \in(\text { ho } \mathbf{S})^{\mathbf{D}}$ is a centric diagram.

(1) If the groups $\lim ^{i+2} \alpha_{i} \bar{X}$ vanish for $i \geq 1$ then at least one realization of $\bar{X}$ exists.

(2) If in addition the groups $\stackrel{\lim }{ }^{i+1} \alpha_{i} \bar{X}$ vanish for $i \geq 1$ then any two realizations of $\bar{X}$ are in the same weak equivalence class.

Remark. See [3, XI, §6] for a discussion of the right derived functors $\varliminf^{i}$ of the inverse limit functor $\lim _{\longleftarrow}: \mathbf{A b}^{\mathbf{D}} \rightarrow \mathbf{A b}$.

Theorem 1.1 is actually a consequence of a sharper and more geometric calculation which is described in $\S 2$.

We study two examples, both involving diagrams that are used to express the classifying space of a compact Lie group as a homotopy direct limit (or at least as a homotopy direct limit up to $\mathrm{Z} / p$ homology). We first show that a slight modification of the centralizer decomposition diagram of [12] is centric. We then show that the $p$-toral decomposition diagram of [13] is centric, and point out that the results of [13] imply that this diagram satisfies the hypotheses of parts (1) and (2) of Theorem 1.1. Then it follows from 1.1(2) that the diagram of [13] is determined up to weak equivalence by its underlying diagram in the homotopy category. 
Organization of the paper. Section 2 contains a discussion of realization complexes and some calculations in the centric case. Theorem 1.1 is proved in $\S 3$. Finally, $\S 4$ deals with the centralizer diagram of [12] and $\S 5$ with the $p$-toral diagram of [13].

Notation and terminology. Some of the simplicial sets used in this note as in [5] are nerves of categories which are not necessarily small. Nevertheless these nerves are homotopically small and it is possible to make standard homotopytheoretic constructions with them.

Since [3, XI] homotopy inverse limits only have homotopy meaning when applied to fibrant diagrams, we sometimes have to replace a given diagram $Y \in \mathbf{S}^{\mathbf{D}}$ by a weakly equivalent fibrant one, such as, $\mathrm{Ex}^{\infty} Y$ where $\mathrm{Ex}^{\infty}$ is the functor of [14]. To simplify notation, we will write $Y^{f}$ instead of $\operatorname{Ex}^{\infty} Y$.

\section{REALIZATION COMPLEXES}

Let $\mathbf{D}$ be a small category, and suppose that $\bar{X}$ is a diagram in (ho $\mathbf{S})^{\mathbf{D}}$. Recall from $[5, \S 3]$ that there is a natural realization complex $r \bar{X}$ associated to $\bar{X}$ such that the components of $r \bar{X}$ are in 1-1 correspondence with weak equivalence classes $\{(Y, f)\}$ of realizations of $\bar{X}$ and such that the component corresponding to a particular $(Y, f)$ is equivalent to the classifying space of an appropriate complex $[5,3.5]$ of homotopy automorphisms of $Y$. The complex $r \bar{X}$ is defined as the nerve of the category whose objects are the realizations of $\bar{X}$ and whose morphisms are weak equivalences between these realizations. Remark 2.4 below indicates that there is a simple characterization of centric maps in terms of realization complexes.

For any object $D$ of $\mathbf{D}$ let $\mathbf{D} \downarrow D$ denote the over category $[3, \mathrm{XI}, \S 2 ; 16$, p. 46] of the identity functor. The objects of this category are pairs $\left(D^{\prime}, f\right)$ where $D^{\prime}$ is an object of $\mathbf{D}$ and $f: D^{\prime} \rightarrow D$ is a map; a morphism $\left(D^{\prime}, f\right) \rightarrow$ $\left(D^{\prime \prime}, g\right)$ is a map $h: D^{\prime} \rightarrow D^{\prime \prime}$ in $\mathbf{D}$ such that $g h=f$. There is a forgetful functor $O_{D}: \mathbf{D} \downarrow D \rightarrow \mathbf{D}$ that maps a pair $\left(D^{\prime}, f\right)$ to $D^{\prime}$. Given a diagram $\bar{X} \in(\text { ho } \mathbf{S})^{\mathbf{D}}$, there is an induced diagram $O_{D}^{*} \bar{X} \in(\text { ho } \mathbf{S})^{\mathbf{D} \downarrow D}$ for each object $D$ of $\mathbf{D}$; there is also a functor $r_{\mathbf{D}^{\text {op }}} X: \mathbf{D}^{\text {op }} \rightarrow \mathbf{S}$ that maps an object $D \in \mathbf{D}^{\text {op }}$ to the realization complex $r\left(O_{D}^{*} \bar{X}\right)$.

The following theorem is proven below:

Theorem 2.1. Suppose that $\mathbf{D}$ is a small category and that $\bar{X}$ is a diagram in ho $\mathbf{S}^{\mathbf{D}}$. Then the natural map $r \bar{X}=\lim ^{\mathbf{D}^{\text {op }}} r_{\mathbf{D}^{\text {op }}} \bar{X} \rightarrow$ ho $\lim ^{\mathbf{D}^{\text {op }}}\left(r_{\mathbf{D}^{\text {op }}} \bar{X}\right)^{f}$ is a weak equivalence.

The main result of this section is a description of the diagram $r_{\mathbf{D}^{\text {op }}} \bar{X}$ in the special case in which $\bar{X}$ is centric.

Theorem 2.2. Suppose that $\mathbf{D}$ is a small category and that $\bar{X} \in(\text { ho } \mathbf{S})^{\mathbf{D}}$ is a centric diagram. Then for each object $D \in \mathbf{D}$ the complex $r_{\mathbf{D}^{\mathrm{op}}} \bar{X}(D)$ has the weak homotopy type of the classifying complex $\bar{W}$ haut $(\bar{X}(D))_{1}$, and for each $i \geq 2$ the functor $\pi_{i}\left(r_{\mathbf{D}^{\mathrm{op}}} \bar{X}\right): \mathbf{D}^{\mathrm{op}} \rightarrow \mathbf{A b}$ is naturally equivalent to $\alpha_{i-1} \bar{X}$.

The rest of this section is taken up with proofs.

Proof of 2.1. For every integer $n \geq 0$ let $\mathbf{n}$ denote the category with the integers $0, \ldots, n$ as objects and with exactly one map $i \rightarrow j$ whenever $i \leq j$. The 
division $d \mathbf{D}$ of $\mathbf{D}$ is the category that has as objects the functors $\mathbf{n} \rightarrow \mathbf{D}$ $(n \geq 0)$ and as maps $\left(J_{1}: \mathbf{n}_{1} \rightarrow \mathbf{D}\right) \rightarrow\left(J_{2}: \mathbf{n}_{2} \rightarrow \mathbf{D}\right)$ the functors $K: \mathbf{n}_{2} \rightarrow$ $\mathbf{n}_{1}$ such that $J_{1} \circ K=J_{2}$. There is a functor $q: d \mathbf{D} \rightarrow \mathbf{D}^{\text {op }}$ given by the formula $(J: \mathbf{n} \rightarrow \mathbf{D}) \mapsto J(n)$. According to [5, 3.7] the natural map $r \bar{X}=$ $\lim ^{d D} r_{d \mathbf{D}} \bar{X} \rightarrow$ ho $\lim ^{d \mathbf{D}}\left(r_{d \mathbf{D}} \bar{X}\right)^{f}$ is a weak equivalence, where $r_{d \mathbf{D}} \bar{X}$ is the functor which assigns to $J: \mathbf{n} \rightarrow \mathbf{D}$ the classification complex $r\left(J^{*} \bar{X}\right)$. Define two objects $q_{\sharp} r_{d \mathbf{D}} \bar{X}$ and $q_{*} r_{d \mathbf{D}} \bar{X}$ of $\mathbf{S}^{\mathbf{D}^{\mathrm{op}}}$ by the formulas

$$
\begin{aligned}
& q_{\sharp} r_{d \mathbf{D}} \bar{X}(D)=\stackrel{D \downarrow q}{\lim } \phi^{*} r_{d \mathbf{D}} \bar{X} \\
& q_{*} r_{d \mathbf{D}} \bar{X}(D)=\operatorname{ho} \underset{D \downarrow q}{\stackrel{\lim }{\longleftarrow} \phi^{*}\left(r_{d \mathbf{D}} \bar{X}\right)^{f},}
\end{aligned}
$$

where $D \downarrow q$ is an under category associated to the functor $q$ [3, XI, §2] and $\phi: D \downarrow q \rightarrow d \mathbf{D}$ is the forgetful functor. There are two things to notice here: first, that the diagram $q_{\sharp} r_{d \mathbf{D}} \bar{X}$ is naturally isomorphic to $r_{\mathbf{D}^{\text {op }}} \bar{X}$ and second, that the natural map $r_{\mathbf{D}}{ }^{\circ \mathrm{p}} \bar{X} \cong q_{\sharp} r_{d \mathbf{D}} \bar{X} \rightarrow q_{*} r_{d \mathbf{D}} \bar{X}$ is a weak equivalence. The first statement follows from inspection, and the second from [5, 3.7] and the fact that for any object $D \in \mathbf{D}^{\text {op }}$ the category $D \downarrow q$ is naturally isomorphic to the division $d(\mathbf{D} \downarrow D)$ in such a way that the functor $\varphi^{*} r_{d \mathbf{D}} \bar{X}$ agrees with the classification functor $r_{d(\mathbf{D} \downarrow D)} O_{D}^{*} \bar{X}$. By $[5,3.7]$ and the pushdown theorem for homotopy inverse limits [6, 9.8] the composite map $r \bar{X} \rightarrow$ ho $\lim ^{d \mathbf{D}}\left(r_{d \mathbf{D}}\right)^{f} \rightarrow$ ho $\lim ^{\mathbf{D}^{\mathrm{op}}} q_{*} r_{d \mathbf{D}} \bar{X}$ is a weak equivalence. The desired result follows immediately:

Lemma 2.3. Suppose that $\mathbf{D}$ is a small category with a terminal object $E$, and that $\bar{X}$ is a centric diagram in $(\text { ho } \mathbf{S})^{\mathbf{D}}$. Let $\mathbf{E}$ be the trivial category with one object and one identity morphism, and $\varepsilon: \mathbf{E} \rightarrow D$ the functor which takes the single object of $\mathbf{E}$ to $E$. Then the map $r \bar{X} \rightarrow r\left(\varepsilon^{*} \bar{X}\right)$ induced by $\varepsilon$ is a weak equivalence.

Proof. We will use some of the notation from the proof of 2.1. First consider the case in which $\mathbf{D}$ is $\mathbf{n}$ for some integer $n$. Let $X_{i}$ be the space $\bar{X}(i), i=$ $1, \ldots, n$, and assume without loss of generality that each $X_{i}$ is fibrant. Let $\gamma_{i}$ be a map $X_{i-1} \rightarrow X_{i}$ that represents the homotopy class $\bar{X}((i-1) \rightarrow(i))$. The argument of $[7,9.3]$ then shows that the classification complex $r \bar{X}$ is naturally weakly equivalent to the bar construction $[7,9.1]$

$$
B\left(\operatorname{haut}\left(X_{0}\right)_{1}, \operatorname{Map}\left(X_{0}, X_{1}\right)_{\gamma_{1}}, \operatorname{haut}\left(X_{1}\right)_{1}, \ldots, \operatorname{haut}\left(X_{n}\right)_{1}\right) \text {. }
$$

A straightforward induction using [7, 9.2(vii)], the definition of the bar construction, and the fact that $\bar{X}$ is centric gives the desired result.

Now assume that $\mathbf{D}$ is an arbitrary small category with a terminal object. The result just proven for the categories $\mathbf{n}, n \geq 0$ shows that the classification diagram $r_{d \mathbf{D}} \bar{X}$ has the property that $r_{d \mathbf{D}} \bar{X}(f)$ is a weak equivalence whenever $f$ is a morphism of $d \mathbf{D}$ such that $q(f)$ is an identity map of $\mathbf{D}^{\mathbf{o p}}$. Let $s d \mathbf{D}$ be the subdivision of $\mathbf{D}[4, \S 5]$; this is the category obtained from $d \mathbf{D}$ by turning all of the "degeneracy maps" (i.e. maps $\left(J_{1}: \mathbf{n}_{1} \rightarrow \mathbf{D}\right) \rightarrow\left(J_{2}: \mathbf{n}_{2} \rightarrow \mathbf{D}\right)$ that correspond to an epimorphism $\left.K: \mathbf{n}_{2} \rightarrow \mathbf{n}_{1}\right)$ into identity maps. The functor $q$ can be factored as a composite $q_{1} \circ q_{0}$, where $q_{0}: d \mathbf{D} \rightarrow s d \mathbf{D}$ is the natural projection and $q_{1}: s d \mathbf{D} \rightarrow \mathbf{D}^{\text {op }}$ is defined like $q$. According to $[6,6.11]$ the 
functor $q_{0}$ is L-cofinal $[6,6.2]$, and according to [4, 5.5 and 5.6] or [6, 6.10(ii)] the functor $q_{1}$ is R-cofinal $[6,6.15]$. Then by [6, 6.5(iv), 6.15] there is some fibrant diagram $Y$ in $\mathbf{S}^{\mathbf{D}^{\text {op }}}$ such that $q^{*}(Y)$ is in the same weak equivalence class as the classification diagram $r_{d \mathbf{D}} \bar{X}$. Moreover, since a functor that is either L-cofinal or R-cofinal is left cofinal $([6,6.6]$ and opposite of $[6,6.7])$ it follows from the cofinality theorem for homotopy inverse limits $[6,9.3]$ that ho $\lim ^{\mathbf{D}^{\text {op }}} Y$ is weakly equivalent to ho $\lim ^{d \mathbf{D}} r_{d \mathbf{D}} \bar{X}$. The lemma now follows in a straightforward way from the fact that $E$ is an initial object of $D^{\text {op }}$, so that the natural map ho $\lim ^{\mathbf{D}^{\text {op }}} Y \rightarrow Y(E)$ is a weak equivalence [3, XI, 4.1(iii)].

Proof of 2.2. The over category $\mathbf{D} \downarrow D$ has a terminal object, so the first assertion is a consequence of Lemma 2.3 and $[5,3.5]$. The second assertion is a routine calculation that uses the bar constructions which appear in the proof of Lemma 2.3; to make this calculation it is enough to restrict to the case in which $\mathbf{D}$ is the category $\mathbf{n}$ for $n=1$.

Remark 2.4. Let $f: A \rightarrow B$ be a map between fibrant simplicial sets and let $\mathbf{0}$ and $\mathbf{1}$ denote the categories $\mathbf{n}$ above for $\dot{h}=0,1$. Consider the functor $f: \mathbf{1} \rightarrow$ ho $\mathbf{S}$ that sends the map $0 \rightarrow 1$ in $\mathbf{1}$ to the homotopy class represented by $f$ and the functor $\bar{B}: 0 \rightarrow$ ho $S$ that sends the object 0 to $B$. It is an immediate consequence of the proof of Lemma 2.3 that $f$ is centric iff the evident realization complex map $r \bar{f} \rightarrow r \bar{B}$ is an equivalence.

\section{Proof OF 1.1}

Given the definition of the realization complex $r \bar{X}$ in $\S 2$, part (1) of Theorem 1.1 comes down to the statement that $r \bar{X}$ is nonempty and part (2) to the statement that $r \bar{X}$ is connected. According to 2.1 the complex $r \bar{X}$ is weakly equivalent to the homotopy inverse limit ho $\lim ^{\mathbf{D}^{\text {op }}}\left(r_{\mathbf{D}^{\text {o }}} \bar{X}\right)^{f}$. Hence $r \bar{X}$ is also weakly equivalent to Tot $Y^{\bullet}$, where $Y^{\bullet}$ is the cosimplicial replacement of $\left(r_{\mathbf{D}^{\mathrm{op}}} \bar{X}\right)^{f}[3, \mathrm{XI}, \S 5]$ and Tot is the "total space" or codiagonal functor [3, X, $\S 3]$. There is a spectral sequence

$$
\left\{E_{2}^{s, t}=\pi^{s} \pi_{t} Y^{\bullet}\right\} \Rightarrow \pi_{t-s} \operatorname{Tot} Y^{\bullet}
$$

that has been studied by Bousfield [1] in great detail, especially in connection with its low-dimensional behavior. It is not necessary to specify basepoints in the above formula for the $E_{2}$-term because, in view of 2.2 and the way in which $Y^{\bullet}$ is constructed [3, XI, 5.1], each space $Y^{s}$ is 1-connected. To work with the spectral sequence beyond $E_{2}$ it is necessary to make successive choices that amount in the limit to choice of a basepoint for Tot $Y^{\bullet}$, but this consideration has been suppressed from the above notation for the abutment of the spectral sequence. By $2.2,[3, \mathrm{XI}, 6.2 ; 1,2.4]$ there are isomorphisms

$$
E_{2}^{s, t}= \begin{cases}* & (s, t)=(0,0) \text { or }(1,1), \\ \{1\} & (s, t)=(0,1), \\ \longleftarrow^{s} \alpha_{t-1} \bar{X} & s \geq 0 \text { and } t \geq 2,\end{cases}
$$

and in fact these are the only cases in which $E_{2}^{s, t}$ is in general defined. Statement (1) of Theorem 1.1 in now a consequence of the next-to-last sentence of 
$[1,6.1]$ in the special case $r=2$, while (2) follows from [1,6.5] in the special case $q=1$.

\section{THE CENTRALIZER DIAGRAM}

Fix a prime number $p$. Let $G$ be a compact Lie group, and let $\mathbf{A}_{G}$ be the category whose objects are the nontrivial elementary abelian $p$-subgroups of $G$; a morphism $A \rightarrow A^{\prime}$ in $\mathbf{A}_{G}$ is a homomorphism $f: A \rightarrow A^{\prime}$ of abelian groups with the property that there exists an element $g \in G$ such that $f(x)=g x g^{-1}$ for all $x \in A$. There is a functor $\hat{\alpha}_{G}$ from $\mathbf{A}_{G}^{\text {op }}$ to the category of topological spaces which sends $A$ to the Borel construction $E G \times_{G}(G / \zeta(A))$, where $\zeta(A)$ denotes the centralizer of $A$ in $G$. (Notice that this Borel construction has the homotopy type of the classifying space $B \zeta(A)$.) The main result of [12] states that the natural map ho $\lim \hat{\alpha}_{G} \rightarrow E G \times_{G} *=B G$ is an isomorphism on $\mathrm{Z} / p$ homology. In this section we will prove that a slight modification of this "centralizer diagram" $\hat{\alpha}_{G}$ is centric. We do not know whether $\hat{\alpha}_{G}$ or its modification satisfy the hypotheses of Theorem 1.1 .

We will tacitly assume in this section as well as in $\S 5$ that the spaces which appear have been replaced by their singular complexes.

For any connected space $X$ let $C X$ denote the partial $\mathrm{Z} / p$-completion of $X$ in the sense of $[3,6.8]$; there is a map $X \rightarrow C X$ that induces an isomorphism on $\mathbf{Z} / p$ homology, an isomorphism on fundamental groups, and the ordinary $\mathbf{Z} / p$-completion map on universal covers. If $G$ is a compact Lie group, then, there are maps $B G \rightarrow C(B G)$ and ho $\lim _{\longrightarrow} C\left(\hat{\alpha}_{G}\right) \rightarrow C(B G)$ which induce isomorphisms on $\mathrm{Z} / p$ homology.

Theorem 4.1. For any compact Lie group $G$ the diagram $C\left(\hat{\alpha}_{G}\right)$ is centric.

In view of the definition of $\hat{\alpha}_{G}$, Theorem 4.1 is an easy consequence of the following more general assertion.

Theorem 4.2. Let $A$ be a finite abelian p-group that is a subgroup of the compact Lie group $G, H$ the centralizer of $A$ in $G$, and $f: C(B H) \rightarrow C(B G)$ the map derived from the inclusion $H \rightarrow G$. Then $f$ is centric.

The proof of 4.2 depends on a number of lemmas.

Lemma 4.3. If $X$ is a 1-connected $\mathrm{Z} /$ p-complete space $[3, \mathrm{I}, \S 5]$ then the homotopy groups of $X$ are uniquely $q$-divisible for every prime $q \neq p$. If $Y$ is a nilpotent space which has the $\mathrm{Z} / p$ homology of a point, then the homotopy groups of $Y$ are uniquely p-divisible.

Proof. The first statement follows from [3, VI, 5.4(ii)]. The second can be proved by a standard induction on the refined Postnikov tower [3, III, 5.3] of $Y$, or by combining the algebraic calculation of the homotopy groups of the $\mathrm{Z} / p$-completion of $Y[3, \mathrm{VI}, 5.1]$ with the fact that this $\mathrm{Z} / p$-completion is contractible if $Y$ has the $\mathrm{Z} / p$ homology of a point.

Lemma 4.4. Let $X$ and $Y$ be connected spaces, $f: X \rightarrow C(Y)$ a map, and $W$ the universal cover of $\operatorname{Map}(X, C(Y))_{f}$. Then $W$ is $\mathbf{Z} / p$-complete.

Proof. Let $g: X \rightarrow K\left(\pi_{1} Y, 1\right)$ be the composite of $f$ with the map $Y \rightarrow$ $K\left(\pi_{1} Y, 1\right)$ which classifies the universal covering fibration $\tilde{Y} \rightarrow Y$. For elementary reasons there is up to homotopy a fibration sequence

$$
\Gamma \rightarrow \operatorname{Map}(X, C(Y))_{f} \rightarrow \operatorname{Map}\left(X, K\left(\pi_{1} Y, 1\right)\right)_{g}
$$


in which $\Gamma$ is a union of components of the space of sections of a certain fibration $E \rightarrow X$ with homotopy fibre $C(\tilde{Y})$. Since the space $\operatorname{Map}\left(X, K\left(\pi_{1} Y, 1\right)\right)$ has no homotopy above dimension 1 , the space $W$ is homotopy equivalent to the universal cover of one component of $\Gamma$. The space $C(Y)$ is $\mathrm{Z} / p$-complete and therefore, $H_{*}(-, \mathbf{Z} / p)$-local in the sense of Bousfield [2, §4]. A slight variation on the construction of $[3, \mathrm{X}, 2.2(\mathrm{ii}), 3.3(\mathrm{i})]$ shows that the space of sections of $E \rightarrow X$ can be expressed as Tot of a fibrant cosimplicial space in which each constituent space has the homotopy type of a product of copies of $C(\tilde{Y})$. Therefore [3, XI, 4.4] the space of sections of $E \rightarrow X$ is equivalent to the homotopy inverse limit of a diagram of $H_{*}(-, \mathrm{Z} / p)$-local spaces and is itself $H_{*}(-, \mathrm{Z} / p)$-local $[2,12.9]$. It follows from [2, 5.5] that the universal cover of any component of $\Gamma$ is $H_{*}(-, \mathbf{Z} / p)$-local and therefore, $[2, \S 4] \mathrm{Z} / p$-complete.

Lemma 4.5. Let $A$ be a finite abelian p-group that is a subgroup of the compact Lie group $G, H$ the centralizer of $A$ in $G$, and $v: B A \cong C(B A) \rightarrow C(B G)$ the map derived from the inclusion $A \rightarrow G$. Then the natural map (see below)

$$
C(B H) \rightarrow \operatorname{Map}(B A, C(B G))_{v}
$$

is an equivalence.

Remark. The multiplication map $A \times H \rightarrow G$ induces a map $B A \times B H \rightarrow B G$ and hence a map $B A \times C(B H) \cong C(B A \times B H) \rightarrow C(B G)$. It is the adjoint of this last map which appears in 4.5.

Proof of 4.5. Let $G_{0}$ be the identity component of $G$ and $F$ the homotopy fibre of the completion map $B G_{0} \rightarrow C\left(B G_{0}\right)$. The space $F$ is simple, because the action of $\pi_{1} F$ on the homotopy groups of $F$ extends to an action of the trivial group $\pi_{1} B G_{0}$. The Serre spectral sequence of the fibration $F \rightarrow B G_{0} \rightarrow$ $C\left(B G_{0}\right)$ immediately shows that $F$ has the $\mathrm{Z} / p$-homology of a point. It follows from Lemma 4.3 that the homotopy groups of $F$ are uniquely $p$-divisible and that $\pi_{1} F$ is $q$-divisible for any prime $q \neq p$. It is clear that $F$ is also the homotopy fibre of the partial completion map $B G \rightarrow C(B G)$.

Let $u: B A \rightarrow B G$ be derived from the inclusion $A \rightarrow G$ and let $F^{\prime}$ be the homotopy fibre of the natural map $U \rightarrow V$, where $U$ is $\operatorname{Map}(B A, B G)_{u}$ and $V$ is $\operatorname{Map}(B A, C(B G))_{v}$. It follows from obstruction theory (cf. [10, proof of 2.3]) that $F^{\prime}$ is connected and that $\pi_{i} F^{\prime}$ is a direct summand of $\pi_{i} F$ for $i \geq 1$; in particular, $\pi_{i} F^{\prime}$ is a uniquely $p$-divisible abelian group for $i \geq 1$ and a divisible abelian group for $i=1$. Consider the exact sequence $\pi_{1} F^{\prime} \rightarrow$ $\pi_{1} U \rightarrow \pi_{1} V \rightarrow\{1\}$. By $[10,1.1]$ the group $\pi_{1} U$ is finite and so cannot accept a nontrivial map from the divisible group $\pi_{1} F^{\prime}$. It follows that $\pi_{1} U \cong \pi_{1} V$, that $F^{\prime}$ is the homotopy fibre of the map $\tilde{U} \rightarrow \tilde{V}$ of universal covers, and hence that $\tilde{U} \rightarrow \tilde{V}$ induces an isomorphism on $\mathbf{Z} / p$ homology. By $[10,1.1]$, then, the natural map $C(B H) \rightarrow V$ induces an isomorphism on fundamental groups and a $\mathbf{Z} / p$ homology equivalence on universal covers. The lemma follows [3, $\mathrm{I}, 5.5]$ from the fact that the universal cover of $C(B H)$ is $\mathrm{Z} / p$-complete by construction, while the universal cover of $V=\operatorname{Map}(B A, C(B G))_{v}$ is $\mathbf{Z} / p$ complete by 4.4 .

Proof of 4.2. Let $K$ be the quotient group $H / A$. By the fibre lemma [3, II] and a short low-dimensional calculation the principal fibration sequence $B A \rightarrow B H \rightarrow B K$ gives rise to an induced principal fibration sequence $B A \rightarrow$ 
$C(B H) \rightarrow C(B K)$. Since the fibre $B A$ in this principal fibration is connected, the monodromy action of $\pi_{1} C(B K)$ on $B A$ is trivial up to homotopy. It follows by elementary homotopy theory that there is an associated fibration $e_{1}: E_{1} \rightarrow C(B K)$ with homotopy fibre $\operatorname{Map}(B A, C(B G))_{v} \quad(v$ is as in Lemma 4.5) such that the space of sections $\Gamma\left(e_{1}\right)$ is naturally homotopy equivalent to the space of maps $C(B H) \rightarrow C(B G)$ which restrict to $v$ on $B A$.

Let $w: B A \rightarrow C(B H)$ be induced by the inclusion $A \rightarrow H$. In a similar way there is a fibration $e_{2}: E_{2} \rightarrow C(B K)$ with homotopy fibre $\operatorname{Map}(B A, C(B H))_{w}$ such that the space of sections $\Gamma\left(e_{2}\right)$ is naturally homotopy equivalent to the space of maps $C(B H) \rightarrow C(B H)$ which restrict to $w$ on $B A$. Composition with $f$ gives a map $E_{2} \rightarrow E_{1}$ over $C(B K)$ which, after passage to spaces of sections and appropriate component selection, induces the precomposition map haut $(C(B H))_{1} \rightarrow \operatorname{Map}(C(B H), C(B G))_{f}$. Now the natural map from the centralizer of $A$ in $H$ to the centralizer of $A$ in $G$ is an isomorphism (both groups are $H$ ); by Lemma 4.5, this implies that the map $\operatorname{Map}(B A, C(B H))_{w} \rightarrow \operatorname{Map}(B A, C(B G))_{v}$ is a homotopy equivalence and hence that the maps $E_{2} \rightarrow E_{1}$ and $\Gamma\left(e_{2}\right) \rightarrow \Gamma\left(e_{1}\right)$ are also homotopy equivalences. This proves the theorem.

\section{THE $p$-TORAL DIAGRAM}

Let $p$ be a fixed prime as in $\S 4$. A compact Lie group $H$ is said to be toral if its connected component $H_{0}$ is a torus; $H$ is $p$-toral if $H_{0}$ is toral and $H / H_{0}$ is a $p$-group. If $G$ is a compact Lie group, $P$ a $p$-toral subgroup of $G$, and $N(P)$ the normalizer of $P$ in $G$, then $P$ is said to be $p$-stubborn if $N(P) / P$ is finite and has no nontrivial normal $p$-subgroups. Let $\mathscr{R}_{p}(G)$ be the full subcategory of the category of $G$-spaces whose objects are the orbits $G / P$ for $p$-stubborn $P \subset G$. There is a functor $\hat{\beta}_{G}$ from $\mathscr{R}_{p}(G)$ to spaces which assigns to $G / P$ the Borel construction $E G \times_{G} G / P \sim B P$. One of the results in [13] states that if $G$ is a compact Lie group the natural map ho $\underline{\lim } \hat{\beta}_{G} \rightarrow E G \times_{G} *=B G$ induces an isomorphism on $\mathrm{Z} / p$ homology.

Theorem 5.1. For any connected compact Lie group $G$ the "p-toral" diagram $\hat{\beta}_{G}$ is centric.

By $[13,2.7,7.1]$, if $G$ is connected the diagram $\pi\left(\hat{\beta}_{G}\right)$ in (hoS $)^{\mathscr{R}_{p}(G)}$ satisfies the hypotheses of parts (1) and (2) of Theorem 1.1. The conclusion of $1.1(1)$ is not interesting in this case, since $\pi\left(\hat{\beta}_{G}\right)$ is already known to have a realization; namely, $\hat{\beta}_{G}$. Statement 1.1(2), however gives the following corollary:

Corollary 5.2. If $G$ is a connected compact Lie group then any realization of the diagram $\pi\left(\hat{\beta}_{G}\right)$ is weakly equivalent, as an object of $\mathbf{S}^{\mathscr{R}_{p}(G)}$, to $\hat{\beta}_{G}$ itself.

This corollary implies that if $G$ is a connected compact Lie group then $B G$ is determined up to $\mathrm{Z} / p$ homology type by a certain relatively small and wellcontrolled diagram in the homotopy category. There is some hope that in the long run this observation will contribute to a generalization of the uniqueness result in [8].

The proof of Theorem 5.1 depends on the following proposition. 
Proposition 5.3. Let $j: K \rightarrow L$ be a map of p-toral compact Lie groups and $H$ the centralizer of $j(K)$ in $L$. Then the natural map (cf. 4.5, $[10, \S 1]$ )

$$
B H \rightarrow \operatorname{Map}(B K, B L)_{B j}
$$

is an equivalence.

Remark. It seems likely that the conclusion of this proposition remains valid under the weaker hypotheses that $K$ is compact Lie and $L$ is toral (see [15]). A slightly modified form of the proposition holds if $K$ is assumed $p$-toral and $L$ is assumed compact Lie [21, 18].

Proof of 5.3. The group $L$ acts on itself by conjugation, and composition of this action with the homomorphism $j$ gives a conjugation action of $K$ on $L$. The fixed point set $L^{K}$ of this action is the centralizer $H$ of $j(K)$ in $L$. As in [10, proof of 4.1], the proposition can be proved by showing that the natural map from $L^{K}$ to the homotopy fixed point set $L^{h K}$ of this conjugation action is an equivalence. (Here $L^{h K}$ is the space of sections of the fibration over $B K$ with fibre $L$ associated to the conjugation action.)

Assume then that $L$ and $K$ are $p$-toral, and that $K$ acts on $L$ via group homomorphisms. We will show that the map $L^{K} \rightarrow L^{h K}$ is an equivalence. We can assume [10, proof of 2.2] that the action of $K$ on the component group $\pi_{0} L$ is trivial, since any component of $L$ that is moved by the action of $K$ contributes neither to $L^{K}$ nor to $L^{h K}$. It is enough, then to show that for each component $L_{i}$ of $L$ the map $L_{i}^{K} \rightarrow L_{i}^{h K}$ is an equivalence. There is no difficulty if $L_{i}^{h K}$ is empty, since then $L_{i}^{K}$ is empty too. Assume then that $L_{i}^{h K}$ is nonempty. It follows that for any finite $p$-subgroup $\tau$ of $K$ the homotopy fixed point set $L_{i}^{h \tau}$ is nonempty and hence by [11] that the fixed point set $L_{i}^{\tau}$ is nonempty. Since $K$ is $p$-toral it is possible to find a dense subgroup $\tau_{\infty}$ of $K$ which is an increasing union $\bigcup_{n} \tau_{n}$ of finite $p$-subgroups; by a compactness argument, then $L_{i}^{\tau_{\infty}}$ is nonempty and hence $L_{i}^{K}$ is nonempty. Pick $x \in L_{i}^{K}$. Multiplication by $x$ then gives a $K$-equivariant homeomorphism between $L_{i}$ and the identity component $L_{0}$ of $L$, so we will be done if we can prove that $L_{0}^{K} \rightarrow L_{0}^{h K}$ is an equivalence. Assume for this purpose that $L$ is connected. The group $L$ is then a torus $\left(S^{1}\right)^{n}$ and the automorphism group of $L$ is the discrete group $\mathrm{GL}(n, \mathbf{Z})$. The identity component of $K$ thus acts trivially on $L$, so that the action of $K$ on $L$ factors through the quotient map $K \rightarrow \pi_{0} K=\sigma$. It is obvious that the natural map $L^{\sigma} \rightarrow L^{K}$ is an isomorphism, and an elementary homotopy theory argument shows that the natural map $L^{h \sigma} \rightarrow L^{h K}$ is an equivalence (this comes down to the fact that the space of sections of the trivial fibration over $B\left(K_{0}\right)$ with fibre $L$ is homotopy equivalent to $L$ itself). Let $\tilde{L}=\mathbf{R}^{n}$ be the universal cover of $L$. The group $\sigma$ acts on the short exact sequence $\{1\} \rightarrow \pi_{1} L \rightarrow \tilde{L} \rightarrow L \rightarrow\{1\}$ and the low-dimensional end of the induced twisted cohomology exact sequence gives isomorphisms $\pi_{0}\left(L^{\sigma}\right) \cong H^{1}\left(\sigma, \pi_{1} L\right), \pi_{1}\left(L^{\sigma}\right) \cong H^{0}\left(\sigma, \pi_{1} L\right)$, and $\pi_{k}\left(L^{\sigma}\right) \cong 0$ for $k \geq 2$. A straightforward calculation now shows that the homotopy groups of $L^{h \sigma}$ are given by the same formulas and that the map $L^{\sigma} \rightarrow L^{h \sigma}$ in fact induces an isomorphism on homotopy.

Proof of 5.1. By $[13,5.1(\mathrm{i})]$ the centralizer in $G$ of a $p$-stubborn $P \subset G$ is the center of $P$. The desired result follows from 5.3. 


\section{REFERENCES}

1. A. K. Bousfield, Homotopy spectral sequences and obstructions, Israel J. Math. 66 (1989), 54-104.

2. __ The localization of spaces with respect to homology, Topology 14 (1975), 133-150.

3. A. K. Bousfield and D. M. Kan, Homotopy limits, completions and localizations, Lecture Notes in Math., vol. 304, Springer-Verlag, Berlin, 1972.

4. W. G. Dwyer and D. M. Kan, Function complexes for diagrams of simplicial sets, Indag. Math. 45 (1983), 139-147.

5. __ Realizing diagrams in the homotopy category by means of diagrams of simplicial sets, Proc. Amer. Math. Soc. 91 (1984), 456-460.

6. _ _ A classification theorem for diagrams of simplicial sets, Topology 23 (1984), 139-155.

7. __, Equivariant homotopy classification, J. Pure Appl. Algebra 35 (1985), 269-285.

8. W. G. Dwyer, H. R. Miller, and C. W. Wilkerson, The homotopic uniqueness of $B S^{3}$ (Algebraic Topology, Barcelona, 1986), Lecture Notes in Math., vol. 1298, Springer-Verlag, Berlin, 1987, pp. 90-105.

9. W. G. Dwyer and C. W. Wilkerson, A cohomology decomposition theorem, Topology (to appear).

10. W. G. Dwyer and A. Zabrodsky, Maps between classifying spaces (Algebraic Topology, Barcelona, 1986), Lecture Notes in Math., vol. 1298, Springer-Verlag, Berlin, 1987, pp. 106-119.

11. S. Jackowski, A fixed-point theorem for p-group actions, Proc. Amer. Math. Soc. 102 (1988), 205-208.

12. S. Jackowski and J. E. McClure, A homotopy decomposition theorem for classifying spaces of compact Lie groups, University of Kentucky, 1988, preprint.

13. S. Jackowski, J. E. McClure, and R. Oliver, Self-maps of classifying spaces, preprint, 1989.

14. D. M. Kan, On c.s.s. complexes, Amer. J. Math. 79 (1957), 449-476.

15. R. Lashof, J. P. May, and G. Segal, Equivariant bundles with abelian structural group, Proceedings of the Northwestern University Conference on Homotopy Theory, Contemporary Math., vol. 19, Amer. Math., Soc., Providence, 1983, pp. 167-176.

16. S. Mac Lane, Categories for the working mathematician, Graduate Texts in Math., vol. 5, Springer-Verlag, Berlin, 1971.

17. J. P. May, Simplicial objects in algebraic topology, Math. Studies No. 11, Van Nostrand, Princeton, 1967.

18. D. Notbohm, Abbildungen zwischen klassifizierenden Räume, Dissertation, Göttingen, 1988.

19. D. G. Quillen, Homotopical Algebra, Lecture Notes in Math., vol. 43, Springer-Verlag, Berlin, 1967.

20. __ Rational homotopy theory, Ann. of Math. 90 (1969), 205-295.

21. A. Zabrodsky, Maps between classifying spaces, unpublished manuscript.

Department of Mathematics, University of Notre Dame, Notre Dame, Indiana 46556

Department of Mathematics, Massachusetts Institute of Technology, Cambridge, MASSACHUSETTS 02139 